\title{
Loop-Mediated Isothermal Amplification for the Rapid Detection of the F200Y Mutant Genotype of Carbendazim-Resistant Isolates of Sclerotinia sclerotiorum
}

Yabing Duan, Ying Yang, Yong Wang, Xiayan Pan, Jian Wu, Yiqiang Cai, Tao Li, Donglei Zhao, Jianxin Wang, and Mingguo Zhou, College of Plant Protection, State \& Local Joint Engineering Research Center of Green Pesticide Invention and Application, Nanjing Agricultural University, Nanjing, 210095, China

\begin{abstract}
Duan, Y., Yang, Y., Wang, Y., Pan, X., Wu, J., Cai, Y., Li, T., Zhao, D., Wang, J., and Zhou, M. 2016. Loop-mediated isothermal amplification for the rapid detection of the F200Y mutant genotype of carbendazim-resistant isolates of Sclerotinia sclerotiorum. Plant Dis. 100:976-983.

The point mutation at codon 200 (TTC $\rightarrow$ TAC, F200Y) confers moderate resistance to carbendazim in Sclerotinia sclerotiorum. This mutant genotype (F200Y) has been detected mainly by determining the minimum inhibitory concentration (MIC), which requires 3 to 5 days. Here, we developed a loop-mediated isothermal amplification (LAMP) assay for the rapid detection of the F200Y mutant genotype of carbendazim-resistant isolates of S. sclerotiorum. Specific LAMP primers were designed and concentrations of LAMP components were optimized. The optimal reaction conditions were 62 to $63^{\circ} \mathrm{C}$ for $45 \mathrm{~min}$. The new LAMP assay requires no special equipment and is highly sensitive and specific (the i.e., it generated positive results with

F200Y mutant genotype but generated negative results with other carbendazim-resistant mutants and with a variety of carbendazim-resistant mutants of Botrytis cinerea and Fusarium graminearum). Inclusion of the loop backward (LB) primer reduced the reaction time to $15 \mathrm{~min}$. Results were identical with LAMP and MIC determinations. The advantages of the LB-accelerated LAMP assay for detection of the F200Y mutant genotype were demonstrated by assaying sclerotia produced on rape stems that were artificially inoculated in the field. The results indicated that the new LAMP assay represents an improved way to detect the F200Y mutant genotype of carbendazim-resistant isolates of $S$. sclerotiorum.
\end{abstract}

Sclerotinia stem rot, caused by Sclerotinia sclerotiorum, is a destructive disease of rape and other crops in many countries, including the United States (Bolton et al. 2006; Purdy 1979), Canada (Bardin and Huang 2001), Australia (Letham et al. 1976), and China (Zhou and Luo 1994). Although this disease is mainly controlled by the application of fungicides (Bardin and Huang 2001; Duan et al. 2013a; Steadman 1979), the extensive use of a single fungicide selects for fungicideresistance in the pathogen. Therefore, resistance detection is important for risk assessment and integrated control of Sclerotinia stem rot.

Since the early 1980 s, the benzimidazole fungicide carbendazim was frequently used to control Sclerotinia stem rot (Ma et al. 2009b). Because carbendazim-resistant populations of S. sclerotiorum have appeared with increasing frequency in rape fields (unpublished data), it is necessary to monitor carbendazim resistance over large regions for effective control of this disease. Previous detection methods have been based on determination of the minimum inhibitory concentration (MIC) (Li et al. 2003; Ma et al. 2009a), which requires substantial time and effort. As an important alternative, molecular methods have been developed for detection of carbendazim-resistant genotypes, and these molecular methods are based on the detection of the mutants that result in resistance. The point mutation at codon 198 $(\mathrm{GAG} \rightarrow \mathrm{GCG}, \mathrm{E} 198 \mathrm{~A})$ or 200 (TTC $\rightarrow$ TAC, F200Y) of the $\beta$-tubulin gene leads to carbendazim resistance in S. sclerotiorum. Although polymerase chain reaction (PCR) has been developed for detecting the E198A mutant genotype (Chen et al. 2009; Li et al. 2002), molecular detection of the F200Y mutant genotype has yet to be reported. Given the importance of Sclerotinia stem rot and of carbendazim resistance, a rapid, economic, and simple technique is needed for detecting the F200Y mutant genotype of carbendazim-resistant isolates of $S$. sclerotiorum.

Loop-mediated isothermal amplification (LAMP) can rapidly amplify nucleic acids with high specificity, sensitivity, and efficiency

Corresponding author: M. Zhou; E-mail: mgzhou@njau.edu.cn

Accepted for publication 9 December 2015.

http://dx.doi.org/10.1094/PDIS-10-15-1185-RE

(C) 2016 The American Phytopathological Society under isothermal conditions (Notomi et al. 2000). The current study describes a LAMP assay for detection of the F200Y mutant genotype of carbendazim-resistant isolates of $S$. sclerotiorum. The new LAMP assay will be useful for assessing carbendazim resistance risk in $S$. sclerotiorum and will also provide important reference data for control of Sclerotinia stem rot of rape or other crops.

\section{Materials and Methods}

Fungal isolates, culture conditions, and reagents. This study used three $S$. sclerotiorum isolates (JY4016, TZ25, and HA61), all of which were obtained from single sclerotia (Table 1). JY4016 is moderately resistant to carbendazim because of a point mutation at codon 200 (Phe to Tyr, F200Y) in the $\beta$-tubulin gene (SS1G_04652); TZ25 is highly resistant to carbendazim because of a point mutation at codon 198 (Glu to Ala, E198A) in the $\beta$-tubulin gene; and HA61 is sensitive to carbendazim. Single-spore isolates of Fusarium graminearum and Botrytis cinerea with moderate or high resistance to carbendazim were also used (Table 1).

The sensitivity of $S$. sclerotiorum to carbendazim was determined on potato dextrose agar medium. Based on their MIC, the tested isolates were divided into three phenotypes: highly resistant, MIC $>100 \mu \mathrm{g} \mathrm{ml}^{-1}$; moderately resistant, $100 \mu \mathrm{g} \mathrm{ml}^{-1}>\mathrm{MIC}>$ $5 \mu \mathrm{g} \mathrm{ml}^{-1}$; and sensitive, MIC $<5 \mu \mathrm{g} \mathrm{ml}^{-1}$.

Carbendazim and other reagents used in this study were the same as in a previous study (Duan et al. 2014c).

DNA extraction. Genomic DNA was extracted from sclerotia of $S$. sclerotiorum or from mycelia of $F$. graminearum and $B$. cinerea using a mixer mill (MM400; Retsh) according to the cetyltrimethylammonium bromide procedure (Duan et al. 2013b; Möller et al. 1992).

Primer design. LAMP primers were designed using Primer explorer V4 software (http://primerexplorer.jp/e/) according to a previous study (Duan et al. 2014c). LAMP primers used in this study are indicated in Table 2 and Figure 1.

Specificity of LAMP primers. To improve the specificity of the LAMP primers, one or two artificial base-pair mismatches were introduced within three nucleotides of the $3^{\prime}$ end of the forward inner primer (FIP) (Table 2). Thus, six sets of LAMP primers (Table 2) were obtained and tested to distinguish the F200Y mutant genotype from the wild type. With the genomic DNA of JY4016 or HA61 as template and with the addition of hydroxynaphthol blue (HNB), LAMP was 
Table 1. Fungal isolates used in this study

\begin{tabular}{|c|c|c|c|c|c|}
\hline Fungus & Isolate & Genotype description & Origin & Resistance phenotype $^{a}$ & LAMP \\
\hline$\overline{\text { Sclerotinia sclerotiorum }}$ & HA61 & Wild type & Jiangsu Province, China & $\mathrm{MBC}^{\mathrm{S}}$ & - \\
\hline S. sclerotiorum & JY4016 & Mutation at codon 200 of $\beta$ tubulin (F200Y) & Jiangsu Province, China & $\mathrm{MBC}^{\mathrm{MR}}$ & + \\
\hline S. sclerotiorum & TZ25 & Mutation at codon 198 of $\beta$ tubulin (E198A) & Jiangsu Province, China & $\mathrm{MBC}^{\mathrm{HR}}$ & - \\
\hline Botrytis cinerea & CGY004 & Mutation at codon 198 of $\beta$ tubulin (E198A) & Jiangsu Province, China & $\mathrm{MBC}^{\mathrm{HR}}$ & - \\
\hline B. cinerea & SD2 & Mutation at codon 198 of $\beta$ tubulin (E198K) & Shandong Province, China & $\mathrm{MBC}^{\mathrm{HR}}$ & - \\
\hline B. cinerea & SD4 & Mutation at codon 198 of $\beta$ tubulin (E198V) & Shandong Province, China & $\mathrm{MBC}^{\mathrm{HR}}$ & - \\
\hline B. cinerea & B20 & Mutation at codon 200 of $\beta$ tubulin (F200Y) & Shandong Province, China & $\mathrm{MBC}^{\mathrm{MR}}$ & - \\
\hline Fusarium graminearum & JT04 & Mutation at codon 198 of $\beta_{2}$ tubulin (E198L) & Anhui Province, China & $\mathrm{MBC}^{\mathrm{HR}}$ & - \\
\hline F. graminearum & $\mathrm{J}-2$ & Mutation at codon 198 of $\beta_{2}$ tubulin (E198K) & Anhui Province, China & $\mathrm{MBC}^{\mathrm{HR}}$ & - \\
\hline F. graminearum & NT7 & Mutation at codon 200 of $\beta_{2}$ tubulin (F200Y) & Anhui Province, China & $\mathrm{MBC}^{\mathrm{MR}}$ & - \\
\hline F. graminearum & R9 & Mutation at codon 167 of $\beta_{2}$ tubulin (F167Y) & Anhui Province, China & $\mathrm{MBC}^{\mathrm{MR}}$ & - \\
\hline
\end{tabular}

${ }^{\mathrm{a}} \mathrm{MBC}^{\mathrm{S}}, \mathrm{MBC}^{\mathrm{MR}}$, and $\mathrm{MBC}^{\mathrm{HR}}$ indicate that the isolate is sensitive, moderately resistant, and highly resistant to MBC, respectively.

b Loop-mediated isothermal amplification: + and - represent positive and negative results, respectively.

Table 2. Primers used in this study

\begin{tabular}{lll}
\hline Primer & \multicolumn{1}{c}{ Sequence $\left(\mathbf{5}^{\prime} \mathbf{-} \mathbf{3}^{\prime}\right)^{\mathbf{a}}$} & Use $^{\mathbf{b}}$ \\
\hline F3 & CTCTCTCTGTTCATCAATTGG & FOP for LAMP \\
B3 & CAACTTTCGGAGATCTGAGT & BOP for LAMP \\
BIP & CTACGGAGATCTTAACCACTTGGTTAAGTTGACCAGGGAAACGGA & BIP for LAMP \\
LB & TCCGCTGTCATGTCCGG & LB primer for LAMP \\
FIP1 & GGTTCTCATGCAAATGTCGTAGAGCGAGAACTCTGACGAGACCTA & FIP F200Y \\
FIP2 & GGTTCTCATGCAAATGTCGTAGAGCGAGAACTCTGACGAGACCAA & FIP F200Y \\
FIP3 & GGTTCTCATGCAAATGTCGTAGAGCGAGAACTCTGACGAGACGTA & FIP F200Y \\
FIP4 & GGTTCTCATGCAAATGTCGTAGAGCGAGAACTCTGACGAGACCTAG & FIP F200Y \\
FIP5 & GGTTCTCATGCAAATGTCGTAGAGCGAGAACTCTGACGAGACCTACA & FIP F200Y \\
FIP6 & GGTTCTCATGCAAATGTCGTAGAGCGAGAACTCTGACGAGACGAA & FIP F200Y \\
Ssbeta383F & TGATGTCGTTCGTCGTGAGGC & Partial fragment \\
Ssbeta383R & GACCAGGGAAACGGAGACAGG & Partial fragment \\
\hline
\end{tabular}

${ }^{a}$ Nucleotides in frames were modified from the sequence of the $\beta$-tubulin gene in the sensitive genotype and the F200Y mutant genotype. Nucleotides in bold are mismatches manually added to specifically detect the F200Y mutant genotype of S. sclerotiorum.

b FOP $=$ forward outer primer, LAMP = loop-mediated isothermal amplification, BOP = backward outer primer, BIP $=$ backward inner primer, $\mathrm{LB}=$ loop backward, FIP = forward inner primer, FIP F200Y = FIP for LAMP detection the F200Y mutant genotype of $S$. sclerotiorum, and Partial fragment $=$ to amplify the partial fragments ( $383 \mathrm{bp}$ ) of the $\beta$-tubulin gene containing the F200Y mutation position.
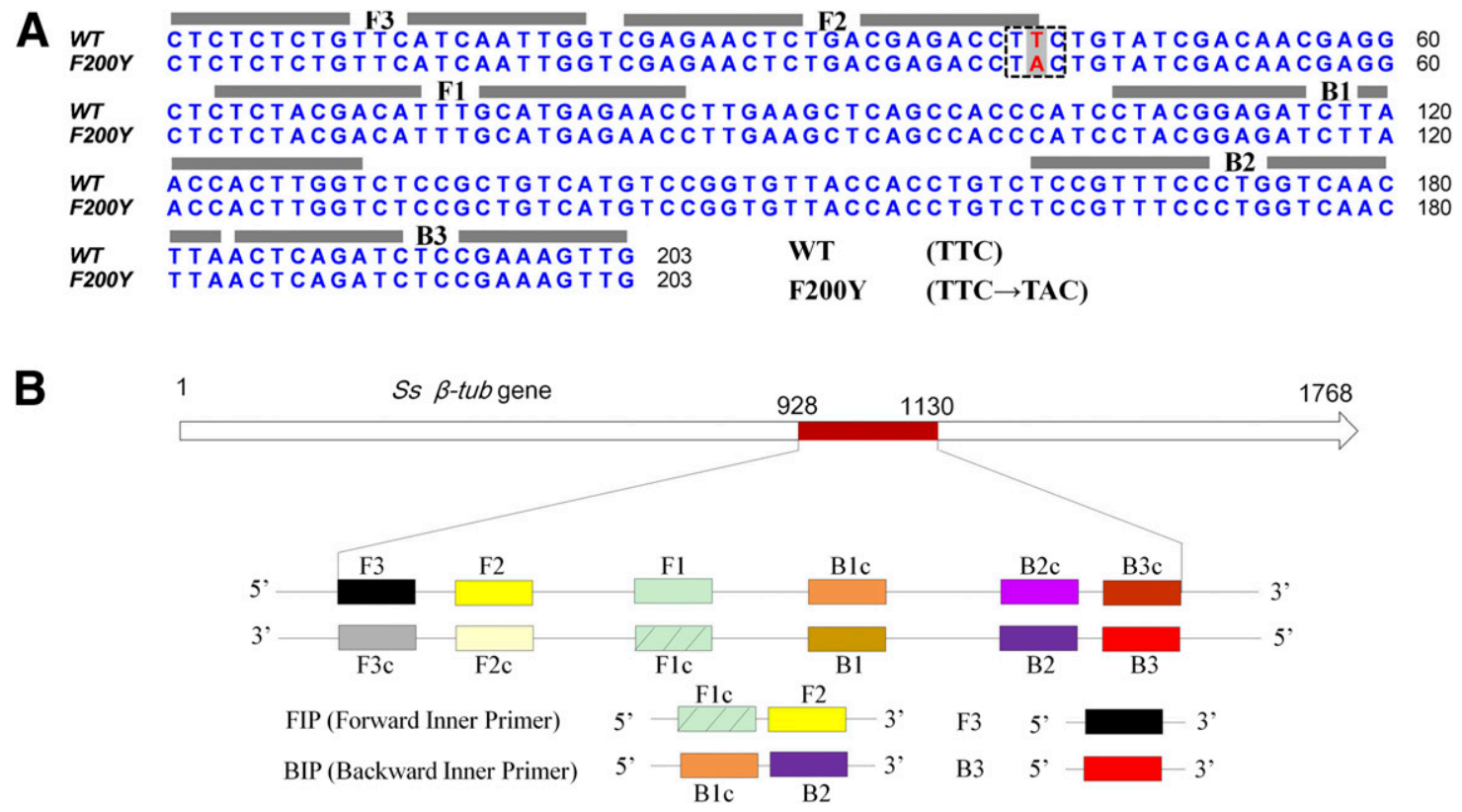

Fig. 1. Schematic illustration of loop-mediated isothermal amplification (LAMP) primers used for detection of F200Y mutant genotype of Sclerotinia sclerotiorum. A, Nucleotide sequence alignment of the target region of the $\beta$-tubulin gene in the wild-type (WT) isolate HA61 and in the moderately carbendazim-resistant isolate JY4016. Sequences used for LAMP primers are indicated by bold lines. The point mutation in the black frame (TTC $\rightarrow$ TAC) leads to a moderate level of carbendazim resistance. $B$, Schematic representation of LAMP primers used in this study. Construction of the inner primers FIP and BIP is shown. F1c and B1c are complementary to F1 and B1, respectively. 
performed at $63^{\circ} \mathrm{C}$ for $60 \mathrm{~min}$ and then at $80^{\circ} \mathrm{C}$ for $10 \mathrm{~min}$ to terminate the reaction. Each product was analyzed by $3.0 \%$ agarose gel electrophoresis; the gels were stained with ethidium bromide and photographed under a UV transilluminator. In addition, the amplification product could also be visually assessed with the unaided eye because HNB caused the color of the mixture in the reaction tube to change from violet to sky blue if the product was present but to remain violet if the product was absent.

Optimization of LAMP reaction components and conditions. As previously described (Duan et al. 2014c), concentrations of LAMP reaction components were optimized in a total volume of $10 \mu \mathrm{l}$ using the optimized LAMP primers and the genomic DNA of S. sclerotiorum JY4016 as template. Then, LAMP reaction temperature and time were also optimized as previously described (Duan et al. 2014c). As before, the results of the LAMP assay were assessed based on both HNB-visualized color change and gel electrophoresis.

Confirmation of the LAMP products. LAMP products were digested in a final volume of $20 \mu \mathrm{l}$ that contained $1 \mu \mathrm{l}$ of $\mathrm{MspI}$ (Takara), $2 \mu l$ of $10 \times \mathrm{T}$ Buffer, $2 \mu l$ of $0.1 \%$ bovine serum albumen, $8 \mu \mathrm{l}$ of LAMP products, and $7 \mu \mathrm{l}$ of double-distilled $(\mathrm{dd}) \mathrm{H}_{2} \mathrm{O}$. The mixture was incubated at $37^{\circ} \mathrm{C}$ overnight, after which the DNA bands were analyzed by $3 \%$ agarose gel electrophoresis; the gels were stained with ethidium bromide and photographed as described earlier. To confirm the identities of the LAMP products, the PCR products were amplified using primers F3 and B3. The PCR mixtures contained $10 \mu \mathrm{M} \mathrm{F3}$ and B3 (2 $\mu$ l of each primer), $2 \mu \mathrm{l}$ of $2.5 \mathrm{mM}$ dNTP, $2.5 \mu \mathrm{l}$ of $10 \times$ PCR buffer $\left(\mathrm{Mg}^{2+}\right.$ free $), 1.5 \mu \mathrm{l}$

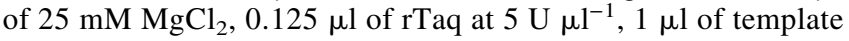
DNA, and $13.875 \mu \mathrm{l}$ of $\mathrm{ddH}_{2} \mathrm{O}$. PCR assays were performed as follows: $94^{\circ} \mathrm{C}$ for $2 \mathrm{~min}$; followed by 35 cycles of denaturation at $94^{\circ} \mathrm{C}$ for $30 \mathrm{~s}$, annealing at $54^{\circ} \mathrm{C}$ for $30 \mathrm{~s}$, and extension at $72^{\circ} \mathrm{C}$ for $30 \mathrm{~s}$; and a final extension at $72^{\circ} \mathrm{C}$ for $10 \mathrm{~min}$. The PCR products were purified by with a gel extraction kit and were cloned into pEASY-T1 vector for sequence analysis.

Specificity of LAMP. The specificity of LAMP was tested with genomic DNA of the fungal isolates or mutants listed in Table 1. The LAMP assay was performed and assessed as described in the previous section and with the optimal LAMP primers and reaction parameters.

Sensitivity of LAMP and PCR. A 383-bp DNA fragment containing the point mutation at codon 200 of the $\beta$-tubulin gene was amplified by PCR using the primers Ssbeta383F and Ssbeta383R. The PCR products were purified and cloned into $\mathrm{pEASY}-\mathrm{T} 1$ vector to create pET383, as described above. The recombinant plasmid pET383 was extracted from positive clones and was 10-fold serially diluted to obtain templates that contained from $1 \times 10^{9}$ to $1 \times 10^{0}$ copies. The templates were subjected to LAMP and PCR. Primers F3 and B3 were used for PCR detection. LAMP was performed as described earlier and with the optimal LAMP primers and reaction parameters.
LAMP detection of $S$. sclerotiorum F200Y mutants from different geographical regions. LAMP was conducted with the genomic DNA of 11 known S. sclerotiorum F200Y mutants from different geographical regions in China (Table 3). Four wild-type isolates and $\mathrm{ddH}_{2} \mathrm{O}$ were used as negative controls.

Acceleration of LAMP by loop primers. To reduce the time required for the LAMP assay, the loop backward (LB) primer was added to the LAMP reaction. LAMP was performed as described earlier with the genomic DNA of JY4016 or HA61 as template.

LAMP field test with artificial inoculation. To evaluate the feasibility of using LAMP to detect the F200Y mutant genotype of carbendazim-resistant mutants of $S$. sclerotiorum, rape stems ('Qinyou number 7') in a field in Jiangsu Province were inoculated with the following mutants or isolates, as previously described (Freeman et al. 2002): four F200Y mutants, three E198A mutants, and three carbendazim-sensitive isolates (Table 4). For each mutant or isolate, one stem on each of three rape plants was inoculated. Multiple sclerotia developed on all inoculated stems. The sclerotia from each stem were divided into two groups; one group was assessed by LAMP (with and without the LB primer) and the other was assessed by MIC determination.

\section{Results}

Specificity of LAMP primers. Based on the point mutation, a LAMP primer set was designed. To determine whether the specificity of this primer could be improved, base pair mismatches were added to the $3^{\prime}$ end of FIP, resulting in six sets of LAMP (Table 2). Genomic

Table 4. Comparison of loop-mediated isothermal amplification (LAMP) and minimum inhibitory concentration (MIC) for detecting the F200Y mutation in carbendazim-resistant isolates of Sclerotinia sclerotiorum in a field test with artificial inoculation

\begin{tabular}{llrrrr}
\hline & & \multicolumn{4}{c}{$\begin{array}{c}\text { Number of positive } \\
\text { samples by }\end{array}$} \\
\cline { 4 - 7 } Isolate & \multicolumn{1}{c}{ Mutation } & $N^{\mathbf{a}}$ & LAMP & LB $^{\mathbf{b}}$ & MIC \\
\hline JH4003 & No mutation & 32 & 0 & 0 & 0 \\
RD4084 & No mutation & 44 & 0 & 0 & 0 \\
NT4070 & No mutation & 36 & 36 & 36 & 0 \\
JY4016 & TTC $\rightarrow$ TAC, Phe to Tyr, F200Y & & 0 & 0 \\
MAS117 & TTC $\rightarrow$ TAC, Phe to Tyr, F200Y & 28 & 28 & 28 & 28 \\
ZG1106 & TTC $\rightarrow$ TAC, Phe to Tyr, F200Y & 22 & 22 & 22 & 22 \\
LA305 & TTC $\rightarrow$ TAC, Phe to Tyr, F200Y & 16 & 16 & 16 & 16 \\
JZ2067 & GAG $\rightarrow$ GCG, Glu to Ala, E198A & 43 & 0 & 0 & 0 \\
NT4082 & GAG $\rightarrow$ GCG, Glu to Ala, E198A & 35 & 0 & 0 & 0 \\
QQ2351 & GAG $\rightarrow$ GCG, Glu to Ala, E198A & 19 & 0 & 0 & 0 \\
\hline
\end{tabular}

a Total number of sclerotia from three replicates.

b Loop backward (LB) primer-accelerated LAMP.

Table 3. Loop-mediated isothermal amplification (LAMP) and minimum inhibitory concentration (MIC) detection of the F200Y mutation in Sclerotinia sclerotiorum isolates obtained from rape fields in different areas in China

\begin{tabular}{lllc}
\hline Isolate & \multicolumn{1}{c}{ Origin } & Mutation of $\boldsymbol{\beta}$-tubulin & LAMP \\
\hline JH4003 & Jianhu, Jiangsu Province & No mutation & - \\
RD4084 & Rudong, Jiangsu Province & No mutation & - \\
NT4070 & Nantong, Jiangsu Province & No mutation & - \\
YZ4018 & Yizheng, Jiangsu Province & No mutation & - \\
JY4016 & Jiangyan, Jiangsu Province & TTC $\rightarrow$ TAC, Phe to Tyr, F200Y & - \\
JK53D5 & Lianyungang, Jiangsu Province & TTC $\rightarrow$ TAC, Phe to Tyr, F200Y & + \\
JY4142 & Jiangyan, Jiangsu Province & TTC $\rightarrow$ TAC, Phe to Tyr, F200Y & + \\
NT1023 & Nantong, Jiangsu Province & TTC $\rightarrow$ TAC, Phe to Tyr, F200Y & + \\
MAS117 & Maanshan, Anhui Province & TTC $\rightarrow$ TAC, Phe to Tyr, F200Y & + \\
ZG1106 & Zhangjiagang, Jiangsu Province & TTC $\rightarrow$ TAC, Phe to Tyr, F200Y & + \\
LA305 & Liuan, Anhui Province & TTC $\rightarrow$ TAC, Phe to Tyr, F200Y & + \\
SY103 & Sheyang, Jiangsu Province & TTC $\rightarrow$ TAC, Phe to Tyr, F200Y & + \\
SZ44 & Suzhou, Jiangsu Province & TTC $\rightarrow$ TAC, Phe to Tyr, F200Y & + \\
JR1309 & Jurong, Jiangsu Province & TTC $\rightarrow$ TAC, Phe to Tyr, F200Y & + \\
XY203 & Xuyi, Jiangsu Province & TTC $\rightarrow$ TAC, Phe to Tyr, F200Y & + \\
\hline
\end{tabular}


DNA of the F200Y mutant genotype and the wild type were used to assess LAMP based on HNB-visualized color change (Fig. 2B) and gel electrophoresis (Fig. 2C). The positive LAMP reaction was indicated by a sky-blue color in tubes with HNB (Fig. 2B) and by a typical ladder-like pattern in gels (Fig. 2C); for negative samples, the tubes remained violet (Fig. 2B) and the gels lacked DNA bands (Fig. 2C). The results indicated that four of the six primer sets (S2, S3, S4, and S5) could be used to detect the F200Y mutant genotype of carbendazim-resistant mutants of $S$. sclerotiorum. The primer set S2 was randomly selected for the further tests in this study.

Optimization of LAMP reaction components. LAMP reaction components were optimized for detection of the F200Y mutant genotype using genomic DNA of JY4016, TZ25, and HA61 as the templates. The best results in terms of HNB-visualized color change (Fig. 3A) and the ladder-like pattern with $3 \%$ gel electrophoresis (Fig. 3B) were obtained when the $10-\mu l$ reaction mixture contained $2.4 \mathrm{U}$ of Bst DNA polymerase, $20 \mathrm{mM}$ Tris- $\mathrm{HCl}, 10 \mathrm{mM} \mathrm{KCl}$, $10 \mathrm{mM}(\mathrm{NH} 4)_{2} \mathrm{SO}_{4}, 0.1 \%$ Triton X-100, $4.5 \mathrm{mM} \mathrm{MgCl} 2,0.8 \mathrm{mM}$ dNTP, $0.64 \mathrm{M}$ betaine, $0.2 \mathrm{mM}$ HNB, $1.2 \mu \mathrm{M}$ each of FIP and BIP, $0.3 \mu \mathrm{M}$ each of F3 and B3, and $1 \mathrm{ng}$ of target DNA.
Restriction enzyme digestion and sequence analysis of the LAMP products. To confirm that the LAMP products were the target fragments, the positive LAMP products were digested with the restriction enzyme $M s p I$ and subjected to gel electrophoresis. The resulting 146- and 57-bp bands (Fig. 3C) were consistent with the bands that were predicted based on the structure of the target fragment. The 203-bp fragment was also amplified by PCR and cloned into the pEASY-T1 vector to create pET203. The plasmid pET203 was transformed into competent cells, and the positive clones were sequenced. The results showed that the 203-bp target fragment was $100 \%$ homologous to the $\beta$-tubulin gene used for primer design.

Optimization of LAMP reaction conditions. Reaction temperature and time were optimized using optimized reaction components and genomic DNA of JY4016 as the template. Color change from violet to sky blue in tubes was observed at 59 to $66^{\circ} \mathrm{C}$ (Fig. 4A) and the ladder-like pattern was observed on gels at 57 to $66^{\circ} \mathrm{C}$ (Fig. 4B) but the bands on gels were dense at 62 to $63^{\circ} \mathrm{C}$ (Fig. 4B). Thus, $62^{\circ} \mathrm{C}$ was selected as the reaction temperature for determining the optimal reaction time. HNB-visualized color change in tubes (Fig. 5A) and the ladder-like pattern in gels (Fig. 5B) were not observed until the

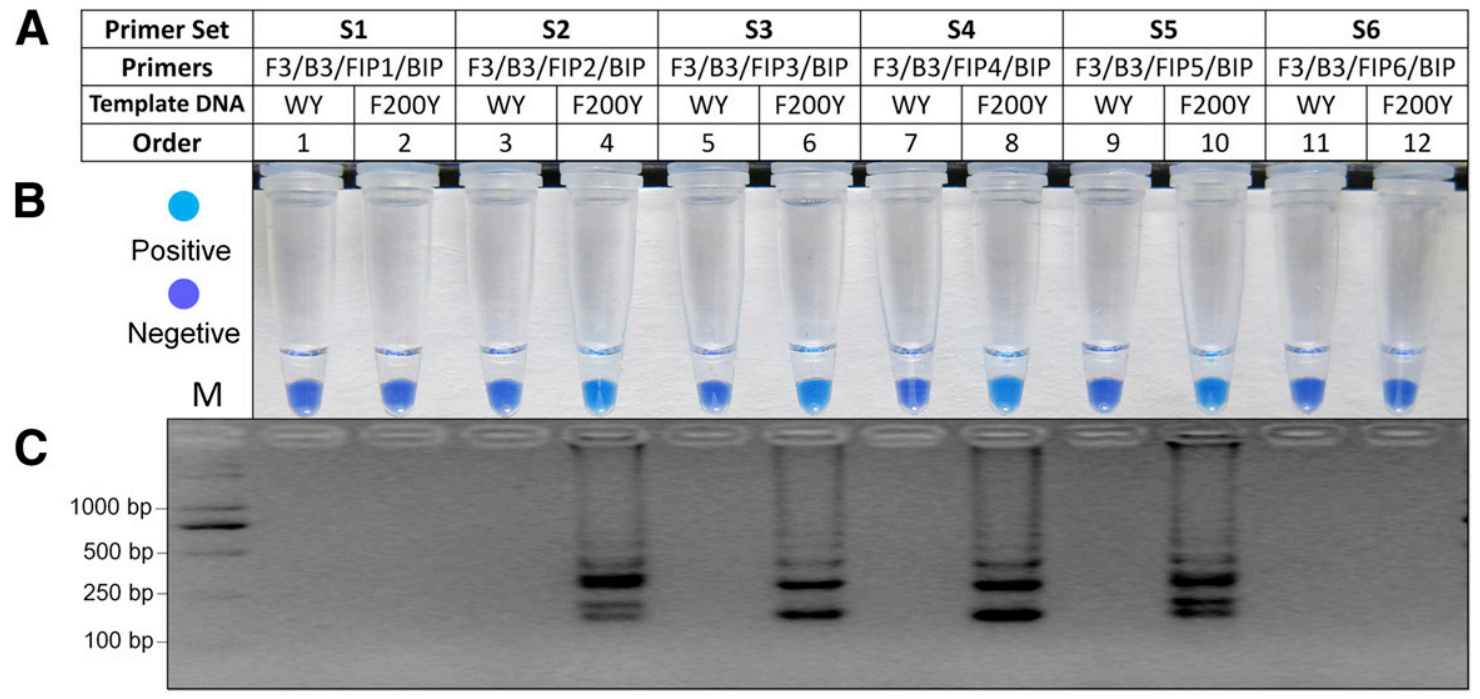

Fig. 2. Optimization of the loop-mediated isothermal amplification (LAMP) primers. A, LAMP primer sets used for optimization. Template DNA was obtained from a Sclerotinia sclerotiorum strain with the F200Y mutation (F200Y) or from a carbendazim-sensitive wild-type strain (WT). Assessment was based on B, hydroxynaphthol blue-visualized color change of the LAMP products and $\mathbf{C}$, gel electrophoresis of the LAMP products.
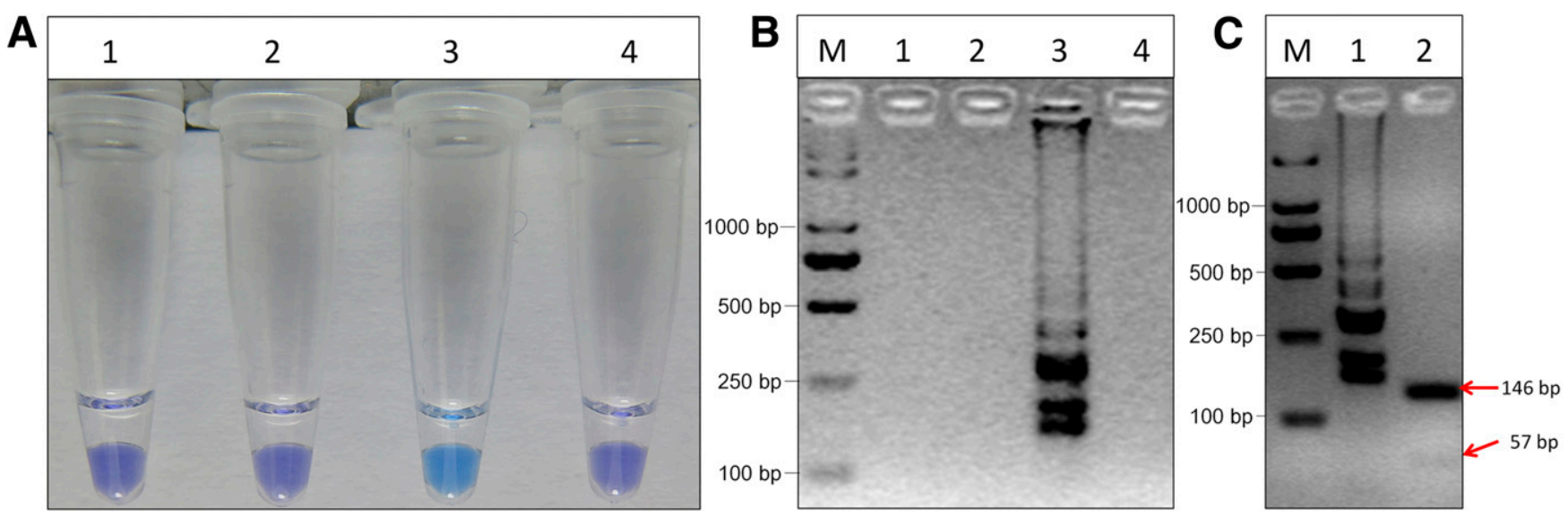

Fig. 3. Loop-mediated isothermal amplification (LAMP) detection of the F200Y mutant genotype in carbendazim-resistant mutants of Sclerotinia sclerotiorum, and digestion of the positive LAMP products. A, LAMP detection as indicated by hydroxynaphthol blue-visualized color change. The reaction becomes sky blue if the $\beta$-tubulin gene has the point mutation at codon 200 but remains violet if the $\beta$-tubulin gene has no mutation or a different mutation. Tube 1, HA61; tube 2, TZ25; tube 3, JY4016; tube 4, double-distilled (dd) $\mathrm{H}_{2} \mathrm{O}$. B, LAMP detection as indicated by gel electrophoresis. The positive reaction is manifested as a ladder-like pattern on the $3.0 \%$ agarose gel. Lane $\mathrm{M}, 2 \mathrm{~K}$ plus; lane 1, HA61; lane 2, TZ25; lane 3, JY4016; lane 4, ddH $\mathrm{H}_{2} \mathrm{O}$. C, LAMP products were digested with Mspl and then subjected to gel electrophoresis. Lane M, 2K plus; lane 1, LAMP products without digestion; lane 2, LAMP products after digestion by Mspl; two fragments (146 and 57 bp) were observed. 
reaction time was increased to $45 \mathrm{~min}$. Therefore, 62 to $63^{\circ} \mathrm{C}$ for $45 \mathrm{~min}$ were selected as the best reaction conditions for LAMP detection of the F200Y mutant genotype of S. sclerotiorum.

Specificity of LAMP. Among the tested fungal isolates or mutants (Table 1), only the F200Y mutant genotype of S. sclerotiorum produced positive results based on HNB-visualized color change (Fig. 6A) and gel electrophoresis (Fig. 6B). This indicated that the established LAMP method had good specificity for detecting the F200Y mutant genotype of carbendazim-resistant mutants of $S$. sclerotiorum.

Sensitivity of LAMP and PCR. To determine the detection limit, PCR and LAMP were performed with 10-fold serial dilutions of the plasmid pET383 as template. Based on HNB-visualized color change

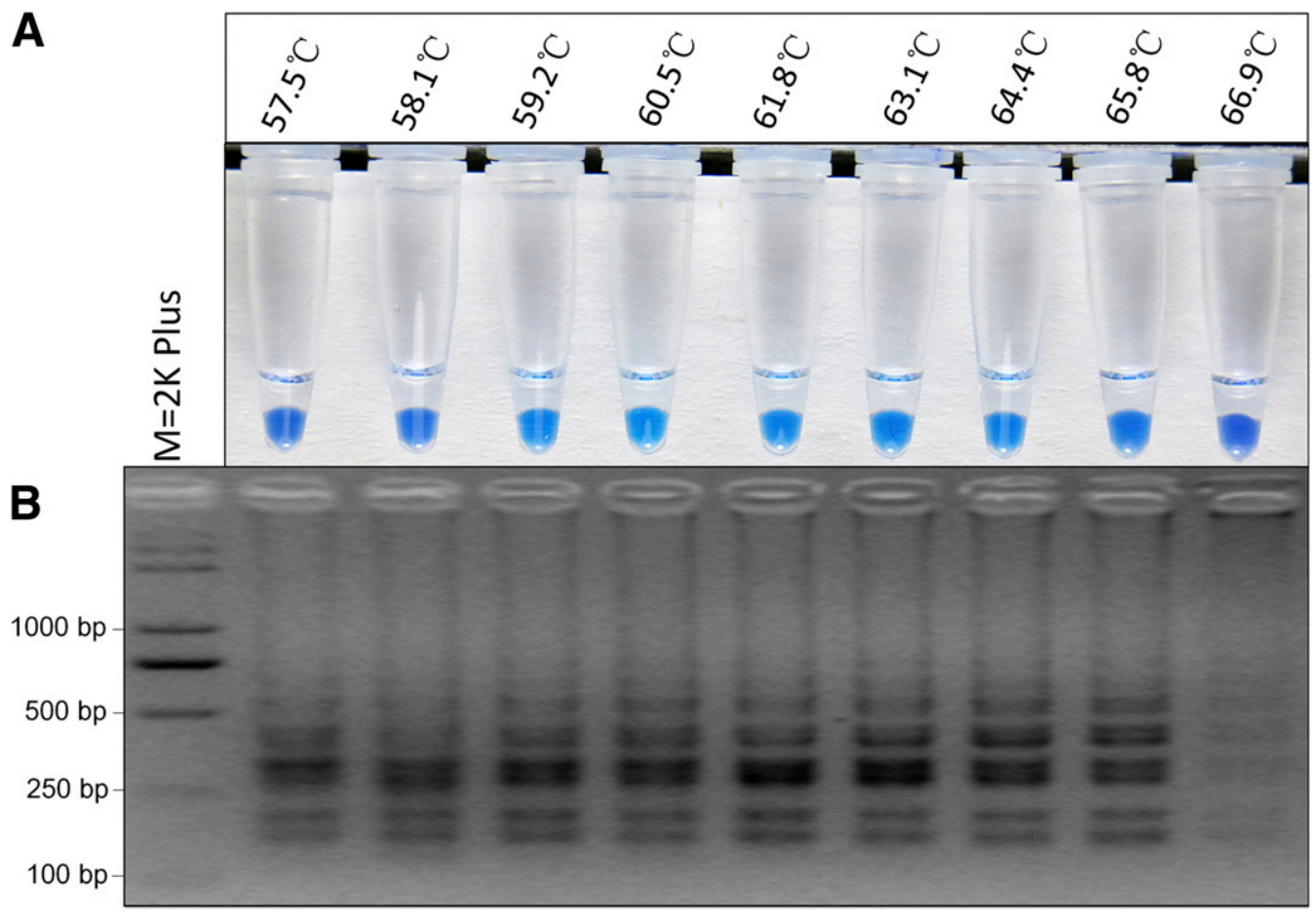

Fig. 4. Optimization of the loop-mediated isothermal amplification (LAMP) reaction temperature for detection of the F200Y mutant genotype in carbendazim-resistant mutants of Sclerotinia sclerotiorum. The experiment used the optimized concentrations of LAMP reaction components, the optimized LAMP primers, and the genomic DNA of S. sclerotiorum JY4016 as template. Assessment based on A, hydroxynaphthol blue-visualized color change and B, gel electrophoresis.

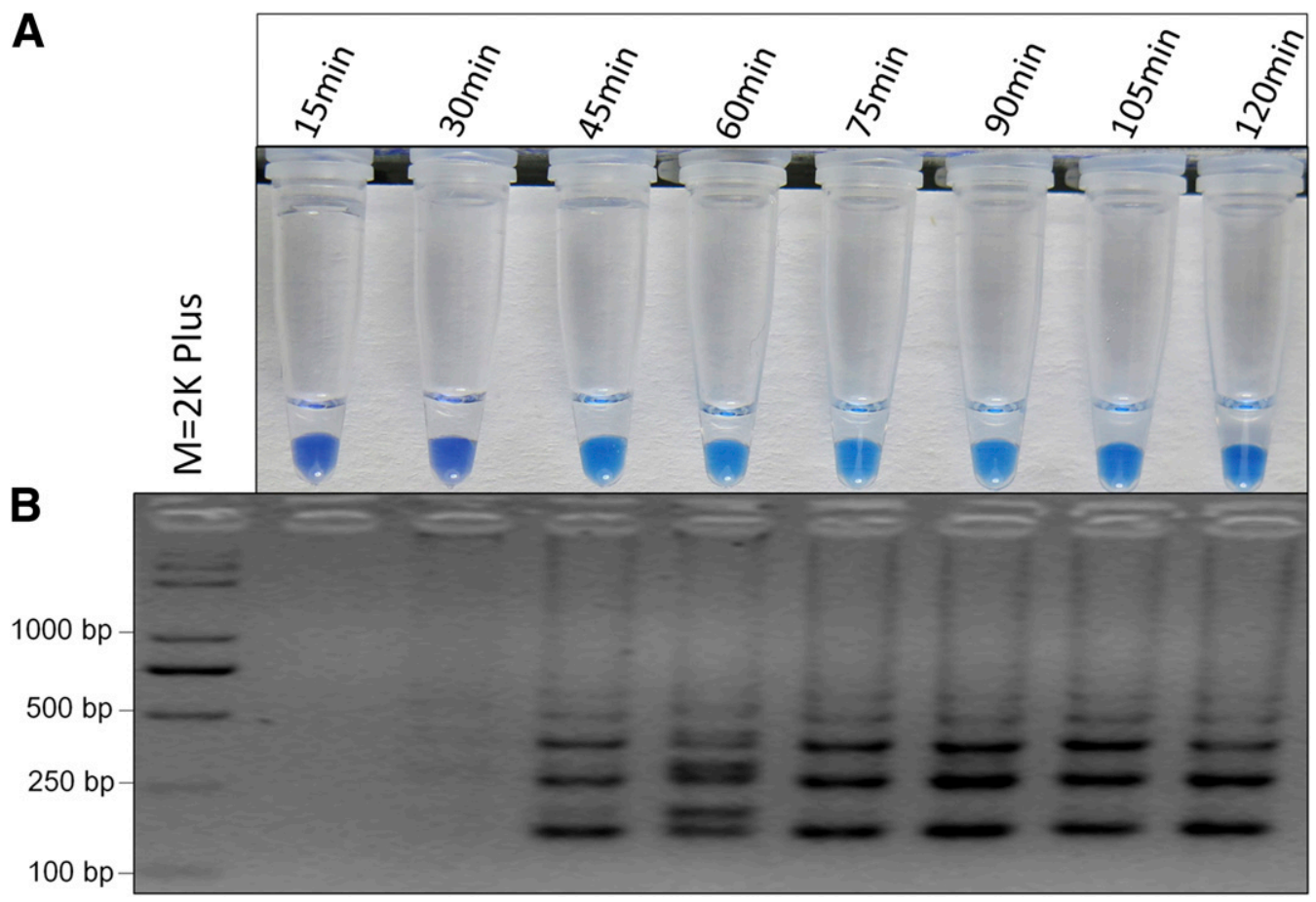

Fig. 5. Optimization of the loop-mediated isothermal amplification (LAMP) reaction time for detection of the F200Y mutant genotype in carbendazim-resistant mutants of Sclerotinia sclerotiorum. The experiment used the optimized concentrations of LAMP reaction components, the optimized LAMP primers and temperature, and the genomic DNA of S. sclerotiorum JY4016 as template. Assessment based on A, hydroxynaphthol blue-visualized color change and B, gel electrophoresis. 
in tubes (Fig. 7A) and the ladder-like pattern in gels (Fig. 7B), the detection limit of the established LAMP assay was $1 \times 10^{2}$ copies. Based on gel electrophoresis, the detection limit of PCR was $1 \times 10^{6}$ copies (Fig. 7C). This indicated that LAMP was 10,000 times more sensitive than PCR.

LAMP detection of $S$. sclerotiorum F200Y mutants from different geographical regions. Before this experiment was conducted, the carbendazim sensitivity of the 15 isolates from different areas was determined (Table 3); based on MIC, 4 of the isolates were sensitive to carbendazim and 11 were resistant. For the resistant isolates, the point mutant genotype at codon 200 in the $\beta$-tubulin gene was confirmed by sequence analysis; the absence of the point mutation was also confirmed for the sensitive isolates. LAMP results were negative for the 4 carbendazim-sensitive isolates and for $\mathrm{ddH}_{2} \mathrm{O}$ but were positive for the $11 \mathrm{~F} 200 \mathrm{Y}$ mutants, as indicated by HNB-visualized color change in tubes (Fig. 8A) and by the ladder-like pattern in gels (Fig. 8B). The results indicated that LAMP could detect S. sclerotiorum F200Y mutants obtained from different fields and areas.

Acceleration of LAMP by loop primers. The LAMP reaction can be accelerated by the addition of two loop primers, termed loop forward (LF) and LB primers (Nagamine et al. 2002). In this study, only the LB primer was designed to accelerate the LAMP reaction

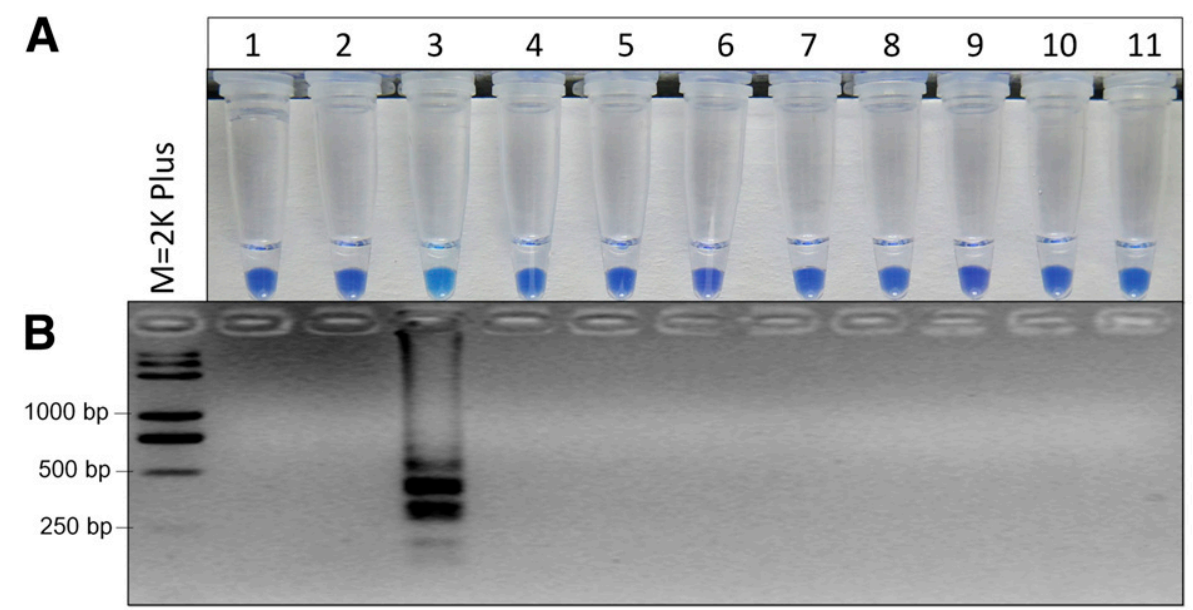

Fig. 6. Specificity of loop-mediated isothermal amplification (LAMP) detection of the F200Y mutant genotype in carbendazim-resistant mutants of Sclerotinia sclerotiorum. Assessment was based on A, hydroxynaphthol blue-visualized color change or B, gel electrophoresis. Lane M, 2K plus; lane 1, wild-type isolate HA61 of $S$. sclerotiorum; lane 2, E198A mutant of S. sclerotiorum; lane 3, F200Y mutant of S. sclerotiorum; lane 4, E198A mutant of Botrytis cinerea; lane 5, E198K mutant of B. cinerea; lane 6, E198V mutant of $B$. cinerea; lane 7, F200Y mutant of $B$. cinerea; lane 8, E198K mutant of Fusarium graminearum; lane 9, E198 L mutant of $F$. graminearum; lane 10, F167Y mutant of $F$. graminearum; lane 11, F200Y mutant of $F$. graminearum.

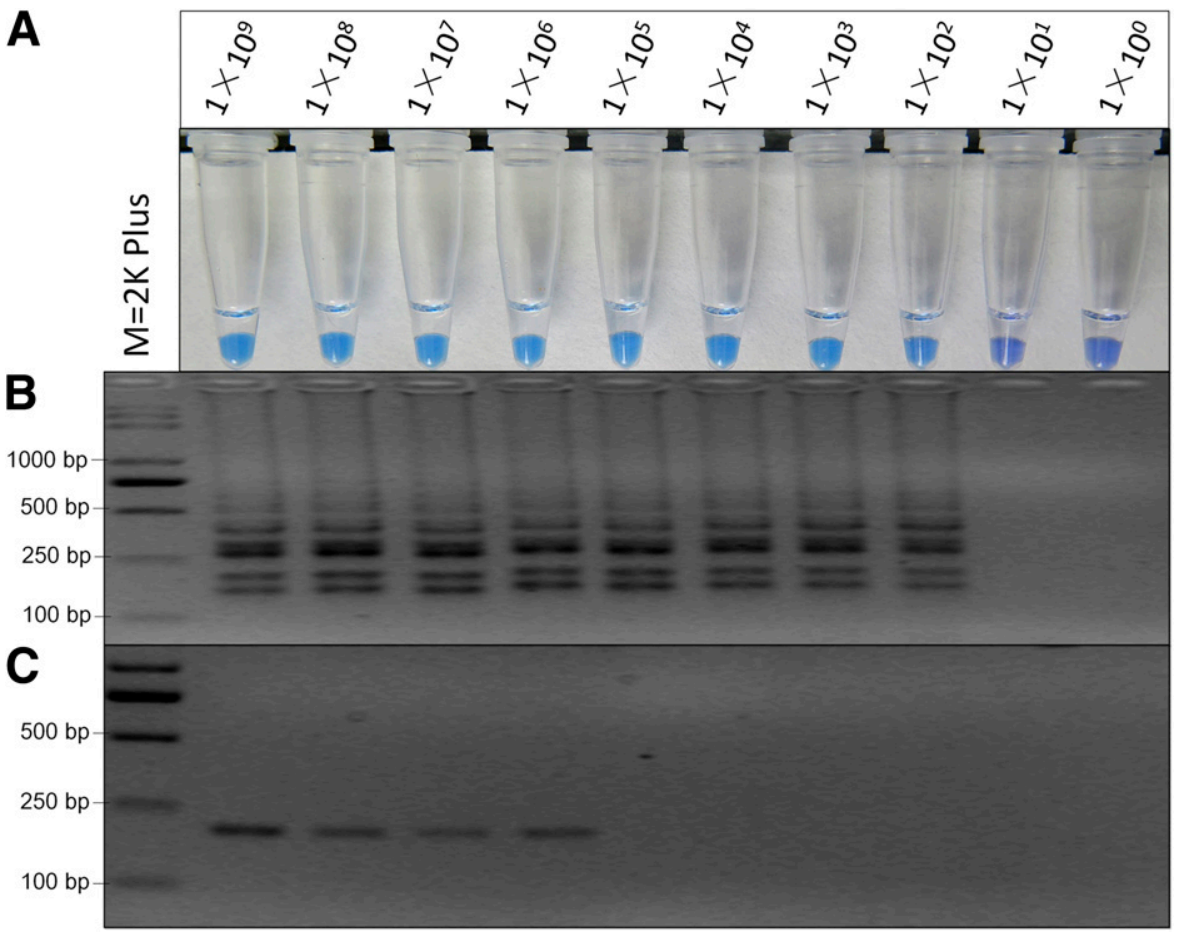

Fig. 7. Sensitivity of loop-mediated isothermal amplification (LAMP) versus conventional polymerase chain reaction (PCR) for detection of the F200Y mutant genotype in carbendazim-resistant mutants of Sclerotinia sclerotiorum. Detection by A, LAMP and hydroxynaphthol blue visualization; B, LAMP and gel electrophoresis; and C, conventional PCR and gel electrophoresis. The template contained the indicated number of copies of the pET383 recombinant plasmid; the pET383 plasmid contained the 383-bp DNA fragment with the F200Y mutation. 
and to distinguish the F200Y mutants from the wild-type isolates. The results indicated that the signal increased at $15 \mathrm{~min}$ with the $\mathrm{LB}$ primer (Fig. 9A and B) rather than at 45 min without the LB primer (Fig. 5A and $\mathrm{B}$ ). Although addition of the LB primer can accelerate the LAMP reaction, the LAMP assay with the LB primer failed to distinguish between F200Y mutants and wild types when the reaction time was increased to $45 \mathrm{~min}$ (Fig. 9A and B). Thus, the reaction time of the LAMP assay should be 15 min when the LB primer is used.

LAMP field test with artificial inoculation. In total, 313 sclerotia of $S$. sclerotiorum were collected from the artificially inoculated rape stems. The sclerotia were divided into two groups. One group was subjected to the LAMP assay (with and without the LB primer), and the other was subjected to the MIC assay. The results for three assays (LAMP, LB-accelerated LAMP, and MIC) were identical (i.e., the assays yielded positive results for sclerotia produced by F200Y mutants and negative results for sclerotia produced by E198A mutants or wildtype isolates) (Table 4).

\section{Discussion}

LAMP is a novel nucleic acid-amplification assay with high specificity and sensitivity, and is also simple to perform. LAMP has been successfully used for the detection of bacterial pathogens (Lee et al. 2015; Niu et al. 2015), viral pathogens (Okuda et al. 2015; Wang et al. 2015), fungal pathogens (Dai et al. 2012; Duan et al. 2014a,b; Niessen and Vogel 2010), and parasites (Kogovšek et al. 2015; Zhuo et al. 2015) in both animals and plants. LAMP has not been used, however, for detecting drug-resistant mutants. In the current study, we developed a LAMP assay for detection of the F200Y mutant genotype of carbendazim-resistant mutants of S. sclerotiorum. To our knowledge, this is the first published study to use LAMP for the detection of F200Y mutants of S. sclerotiorum.

LAMP is performed isothermally without a thermal cycler (Notomi et al. 2000), and the results can be visually assessed based on turbidity (Patel et al. 2015) or by adding DNA-intercalating dyes or metal-ion indicators such as SYBR green (Parida et al. 2005), Picogreen (Curtis et al. 2008), HNB (Goto et al. 2009), $\mathrm{CuSO}_{4}$ (Zoheir and Allam 2011), or calcein (Tomita et al. 2008). Turbidity detection requires expensive equipment, which is not suitable for small laboratories. DNAintercalating dyes are usually added after the reaction is complete, and such addition can easily result in contamination. The risk of contamination is reduced by adding the metal-ion indicator HNB before amplification and by keeping the reaction tubes closed. In HNBvisualized detection, positive samples change from violet to sky blue while negative samples remain violet. LAMP results can also be assessed by gel electrophoresis; positive samples generate a ladderlike pattern in the agarose gel.

To guarantee the specificity of the LAMP primers developed in this study, we tested six sets of LAMP primers, and four of these primer sets distinguished between $S$. sclerotiorum strains with and without the F200Y mutation. Although any one of these four sets of primers could probably be used, we randomly selected S2 as the primer set to be used for optimization of LAMP reaction components and conditions and for further study of assay performance. Once component concentrations were optimized, we found that the optimal LAMP conditions were 62 to $63^{\circ} \mathrm{C}$ for $45 \mathrm{~min}$.

Nagamine et al. (2002) reported that the LAMP reaction could be accelerated by the addition of LF and LB primers. To accelerate the LAMP reaction for detection of the F200Y mutant genotype of S. sclerotiorum, we designed an LB primer. The addition of this primer reduced the LAMP reaction time from 45 to $15 \mathrm{~min}$. However, if the assay was run in the presence of the LB primer for $45 \mathrm{~min}$, it failed to distinguish between an F200Y mutant and a wild-type isolate. Thus, the reaction should keep at $15 \mathrm{~min}$ if the LB primer is used.

We tested the specificity of the LAMP assay developed here with DNA extracted from different carbendazim-resistant mutants of $S$. sclerotiorum, B. cinerea, and $F$. graminearum whose resistance was based on various point mutations in the $\beta$-tubulin gene. The assay was specific in that it generated positive results only for the F200Y mutants in S. sclerotiorum. The new LAMP assay also generated positive results for all $11 \mathrm{~S}$. sclerotiorum F200Y mutants that were obtained from different fields in different areas of China. The detection limits of the new LAMP assay and conventional PCR were compared using serial 10-fold dilutions of plasmid pET383 as templates; the plasmid

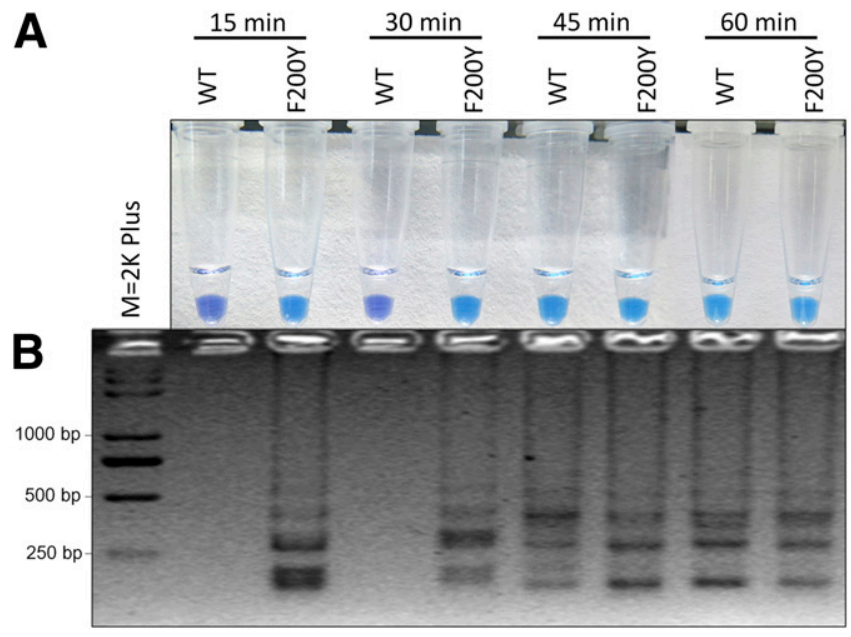

Fig. 9. Acceleration of loop-mediated isothermal amplification (LAMP) by the loop backward primer for the detection of the F200Y mutant genotype in carbendazimresistant mutants of Sclerotinia sclerotiorum. Template DNA was obtained from a Sclerotinia sclerotiorum strain with the F200Y mutation (F200Y) or from a carbendazimsensitive wild-type strain (WT). Assessment based on A, hydroxynaphthol bluevisualized color change or $\mathbf{B}$, gel electrophoresis.

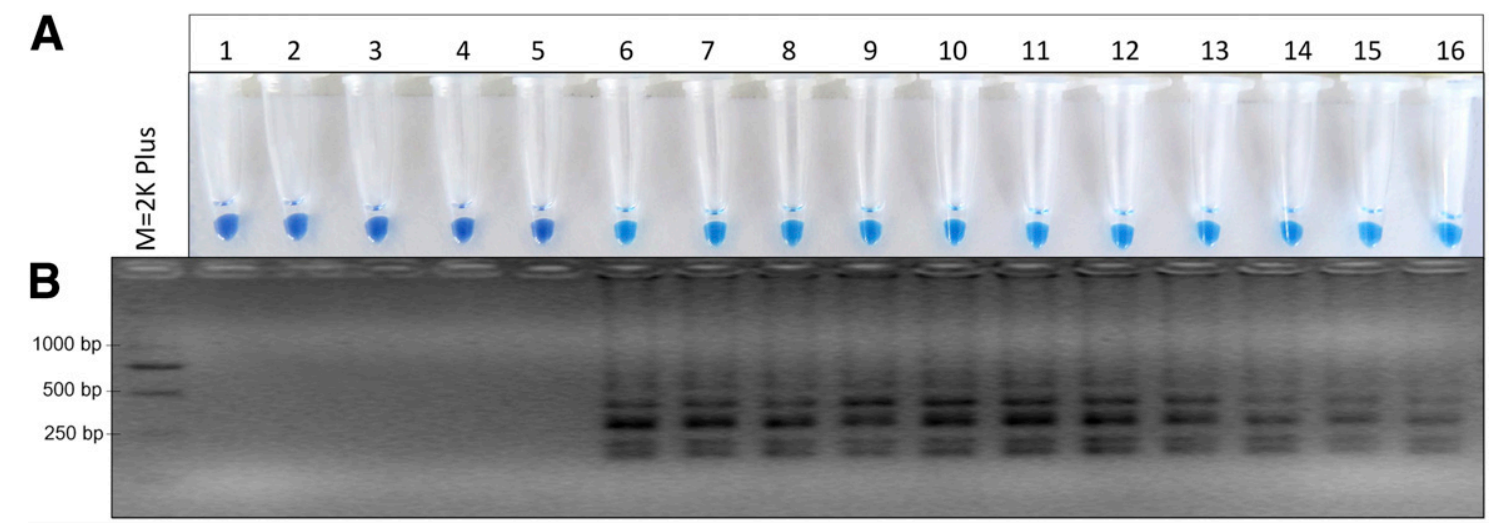

Fig. 8. Loop-mediated isothermal amplification (LAMP) detection of Sclerotinia sclerotiorum F200Y mutants from different geographical regions. Assessment based on A, hydroxynaphthol blue visualized color change or $\mathbf{B}$, gel electrophoresis. Lane 1, double-distilled $\mathrm{H}_{2} \mathrm{O}$; lanes 2 to 5 , wild-type isolates; lanes 6 to 16 , known F200Y mutants. The order of isolates and mutants (from left to right) is the same as in Table 3 (from top to bottom). 
contained a DNA fragment with the F200Y mutation. The new LAMP assay was 10,000 times more sensitive than conventional PCR.

The detection of carbendazim-resistant mutants of S. sclerotiorum by MIC often requires 2 to 5 days. Although PCR requires only 4 to $5 \mathrm{~h}$, it requires expensive equipment, including a PCR thermocycler, an electrophoresis set, and a gel-documentation system. In addition, the PCR assay exposes researchers to toxic materials or conditions, including ethidium bromide, gel-red, and ultraviolet light. The LAMP assay greatly reduces the detection time and requires only a heating block, which is available in most laboratories. The results of the LAMP assay can be visually assessed without special or expensive equipment.

To assess the application of LAMP for detecting the F200Y mutant genotype in carbendazim-resistant mutants of $S$. sclerotiorum in the field, we artificially inoculated rape stems in a field with a mutant and wild-type isolate and subjected the sclerotia that developed to the three assay methods: LAMP, LB-accelerated LAMP, and MIC. The results were identical for all three assay methods, while the LAMP assay was easier to perform than the MIC assay and the LB-accelerated LAMP was the most rapid.

Therefore, we conclude that the LB-accelerated LAMP assay described here will be useful for the detection of the F200Y mutant genotype of carbendazim-resistant mutants of $S$. sclerotiorum. Although the current study reported on $S$. sclerotiorum isolates exclusively from rape fields, our unpublished data indicate that the LB-accelerated LAMP assay will be useful for isolates obtained from any crop.

\section{Acknowledgments}

This research was supported by the National Natural Science Foundation of China (31401764), the Fundamental Research Funds for the central Universities (KJQN201508), the Youth Foundation of Jiang'su Scientific Committee (BK20140679), the Special Fund for Agro-scientific Research in the Public Interest (201303023), and the Students' Research Training Plan Program (1512A01).

\section{Literature Cited}

Bardin, S. D., and Huang, H. C. 2001. Research on biology and control of Sclerotinia diseases in Canada. Can. J. Plant Pathol. 23:88-98.

Bolton, D. M., Thomma, P. H. J. B., and Nelson, D. B. 2006. Sclerotinia sclerotiorum (Lib.) de Bary: Biology and molecular traits of a cosmopolitan pathogen. Mol. Plant Pathol. 7:1-16.

Chen, C. J., Zhao, W., Lu, Y. J., Wang, J. X., Chen, Y., Li, H. X., and Zhou, M. G. 2009. High-throughput detection of highly benzimidazole-resistant allele E198A with mismatch primers in allele-specific real-time polymerase chain reaction. Pest Manage. Sci. 65:413-419.

Curtis, K. A., Rudolph, D. L., and Owen, S. M. 2008. Rapid detection of HIV-1 by reverse transcription, loop-mediated isothermal amplification (RT-LAMP). J. Virol. Methods 151:264-270.

Dai, T. T., Lu, C. C., Lu, J., Dong, S., Ye, W., Wang, Y., and Zheng, X. 2012. Development of a loop-mediated isothermal amplification assay for detection of Phytophthora sojae. FEMS Microbiol. Lett. 334:27-34.

Duan, Y. B., Ge, C. Y., Liu, S. M., Chen, C. J., and Zhou, M. G. 2013a. Effect of phenylpyrrole fungicide fludioxonil on morphological and physiological characteristics of Sclerotinia sclerotiorum. Pestic. Biochem. Physiol. 106:61-67.

Duan, Y. B., Ge, C. Y., Liu, S. M., Wang, J. X., and Zhou, M. G. 2013b. A two component histidine kinase $S h k 1$ controls stress response, sclerotial formation and fungicide resistance in Sclerotinia sclerotiorum. Mol. Plant Pathol. 14:708-718.

Duan, Y. B., Ge, C. Y., Zhang, X. K., Wang, J. X., and Zhou, M. G. 2014a. A rapid detection method for the plant pathogen Sclerotinia sclerotiorum based on loopmediated isothermal amplification (LAMP). Australas. Plant Pathol. 43:61-66.

Duan, Y. B., Ge, C. Y., Zhang, X. K., Wang, J. X., and Zhou, M. G. 2014b. Development and evaluation of a novel and rapid detection assay for Botrytis cinerea based on loop-mediated isothermal amplification. PLoS One 9:e111094.

Duan, Y. B., Zhang, X. K., Ge, C. Y., Wang, Y., Cao, J. H., Jia, X. J., Wang, J. X., and Zhou, M. G. 2014c. Development and application of loop-mediated isothermal amplification for detection of the F167Y mutation of carbendazimresistant isolates in Fusarium graminearum. Sci. Rep. 4:7094.

Freeman, J., Ward, E., Calderon, C., and McCartney, A. 2002. A polymerase chain reaction (PCR) assay for the detection of inoculum of Sclerotinia sclerotiorum. Eur. J. Plant Pathol. 108:877-886.
Goto, M., Honda, E., Ogura, A., Nomoto, A., and Hanaki, K. 2009. Colorimetric detection of loop-mediated isothermal amplification reaction by using hydroxynaphthol blue. Biotechniques 46:167-172.

Kogovšek, P., Hodgetts, J., Hall, J., Prezelj, N., Nikolić, P., Mehle, N., Lenarčič, R., Rotter, A., Dickinson, M., Boonham, N., Dermastia, M., and Ravnikar, M. 2015. LAMP assay and rapid sample preparation method for on-site detection of flavescence doree phytoplasma in grapevine. Plant Pathol. 64:286-296.

Lee, D., Kim, E. J., Kilgore, P. E., Kim, S. A., Takahashi, H., Ohnishi, M., Anh, D. D. Dong, B. Q., Kim, J. S., Tomono, J., Miyamoto, S., Notomi, T., Kim, D. W., and Seki, M. 2015. Clinical evaluation of a loop-mediated isothermal amplification (LAMP) assay for rapid detection of Neisseria meningitidis in cerebrospinal fluid. PLoS One 10:e0122922. doi:10.1371/journal.pone.0122922

Letham, D. B., Huett, D. O., and Trimboli, D. S. 1976. Biology and control of Sclerotinia sclerotiorum in cauliflower and tomato crops in coastal New South Wales. Plant Dis. Rep. 60:286-289.

Li, H. X., Lu, Y. J., Zhou, M. G., and Wang, X. F. 2003. Mutation in $\beta$-tubulin of Sclerotinia sclerotiorum conferring resistance to carbendazim in rapeseed field isolate. Chin. J. Oil Crop Sci. 25:56-60.

Li, H. X., Zhou, M. G., and Lu, Y. J. 2002. Using polymerase chain reaction for detection of carbendazim resistance in Sclerotinia sclerotiorum. Mycosystema 21:370-374.

Ma, H. X., Chen, Y., Wang, J. X., Yu, W. Y., Tang, Z. H., Chen, C. J., and Zhou, M. G. 2009a. Activity of carbendazim, dimethachlon, iprodione, procymidone and boscalid against Sclerotinia stem rot in Jiangsu Province of China Phytoparasitica 37:421-429.

Ma, H. X., Feng, X. J., Chen, Y., Chen, C. J., and Zhou, M. G. 2009b. Occurrence and characterization of dimethachlon insensitivity in Sclerotinia sclerotiorum in Jiangsu Province of China. Plant Dis. 93:36-42.

Möller, E. M., Bahnweg, G., Sandermann, H., and Geiger, H. H. 1992. A simple and efficient protocol for isolation of high molecular weight DNA from filamentous fungi, fruit bodies, and infected plant tissues. Nucleic Acids Res. 20:6115-6116.

Nagamine, K., Hase, T., and Notomi, T. 2002. Accelerated reaction by loopmediated isothermal amplification using loop primers. Mol. Cell. Probes 16: 223-229.

Niessen, L., and Vogel, R. F. 2010. Detection of Fusarium graminearum DNA using a loop-mediated isothermal amplification (LAMP) assay. Int. J. Food Microbiol. 140:183-191.

Niu, J. H., Gao, Y. R., Yin, J. M., Leng, Q. Y., Yang, G. S., Wang, C., and Ren, Y. 2015. Development and evaluation of a loop-mediated isothermal amplification assay for rapid detection of bacterial blight pathogen (Xanthomonas axonopodis pv. dieffenbachiae) in anthurium. Eur. J. Plant Pathol. 142:801-813.

Notomi, T., Okayama, H., Masubuchi, H., Yonekawa, T., Watanabe, K., Amino, N., and Hase, T. 2000. Loop-mediated isothermal amplification of DNA. Nucleic Acids Res. 28:e63

Okuda, M., Okuda, S., and Iwai, H. 2015. Detection of Cucurbit chlorotic yellows virus from Bemisia tabaci captured on sticky traps using reverse transcription loop-mediated isothermal amplification (RT-LAMP) and simple template preparation. J. Virol. Methods 221:9-14.

Parida, M., Horioke, K., Ishida, H., Dash, P. K., Saxena, P., Jana, A. M., Islam, M. A., Inoue, S., Hosaka, N., and Morita, K. 2005. Rapid detection and differentiation of dengue virus serotypes by a real-time reverse transcription-loop-mediated isothermal amplification assay. J. Clin. Microbiol. 43:2895-2903.

Patel, J. S., Brennan, M. S., Khan, A., and Ali, G. S. 2015. Implementation of loopmediated isothermal amplification methods in lateral flow devices for the detection of Rhizoctonia solani. Can. J. Plant Pathol. 37:118-129.

Purdy, L. H. 1979. Sclerotinia sclerotiorum: History, diseases and symptomatology, host range, geographic distribution and impact. Phytopathology 69:875-880.

Steadman, J. R. 1979. Control of plant diseases caused by Sclerotinia species. Phytopathology 69:904-907.

Tomita, N., Mori, Y., Kanda, H., and Notomi, T. 2008. Loop-mediated isothermal amplification (LAMP) of gene sequences and simple visual detection of products. Nat. Protoc. 3:877-882.

Wang, X., Li, X., Hu, S., Qu, H., Zhang, Y., Ni, H., and Wang, X. 2015. Rapid detection of active human cytomegalovirus infection in pregnancy using loop-mediated isothermal amplification. Mol. Med. Rep. 12:2269-2274.

Zhou, B. W., and Luo, Q. 1994. Page 346 in: Rapeseed Diseases and Control. China Commerce Publishing Co., Beijing.

Zhuo, X., Huang, B., Luo, J., Yu, H., Yan, B., Yang, Y., and Du, A. 2015 Development and application of loop-mediated isothermal amplification assays based on ITS-1 for rapid detection of Toxoplasma gondii in pork. Vet. Parasitol. 208:246-249.

Zoheir, K. M., and Allam, A. A. 2011. A rapid improved method for sexing embryo of water buffalo. Theriogenology 76:83-87. 